\title{
Anestesia espinal parte V. Efectos fisiológicos
}

\section{Spinal anesthesia part V. Physiological effects}

\author{
Héctor J. Lacassie ${ }^{1}$, Juan Carlos De La Cuadra F. ${ }^{1}$, Catalina Kychenthal L. ${ }^{2}$, María Jesús Irarrázaval M. ${ }^{2}$, Fernando Altermatt C. 1,* \\ 1 División de Anestesiología, Escuela de Medicina, Pontificia Universidad Católica de Chile. Santiago, Chile. \\ 2 Escuela de Medicina, Pontificia Universidad Católica de Chile. Santiago, Chile.
}

Financiamiento: fondos divisionales.

No se declaran conflictos de interés.

Fecha de recepción: 22 de octubre de 2020 / Fecha de aceptación: 01 de noviembre de 2020

\begin{abstract}
Spinal anesthesia is a widely used technique in the intraoperative setting. It produces characteristic physiological effects on different body systems, which are essential to consider when using this anesthetic method. These effects may be particularly relevant in certain patients depending on their needs, comorbidities and the procedure they will be subjected to. This review highlights the major effects that spinal anesthesia has on the main body systems. In the autonomic nervous system, the effects will depend on the balance and regulation of the sympathetic and parasympathetic systems, secondary to the block level achieved, which can be estimated through temperature discrimination. The sympathetic blockade produced leads to systemic arterial and venous vasodilation, causing a decrease in peripheral vascular resistance, venous return, heart rate and myocardial contractility. Spinal anesthesia also affects the central nervous system in many ways, resulting in a sedative effect. In normal conditions, ventilatory function is not significantly affected, but may become insufficient or unsatisfactory in certain patient groups. Due to blood flow redistribution, thermoregulation may become compromised, decreasing the patient's body temperature according to the degree of the effect. Other organs which may become affected by nervous system regulations are the ones on the gastrointestinal and urinary systems. In addition to this, eventual adrenal blockade could result in lower metabolic and stress responses to stimuli.
\end{abstract}

Key words: Anesthesia, regional anesthesia, spinal anesthesia, physiology.

\section{RESUMEN}

La anestesia espinal es una técnica ampliamente utilizada hoy en día en contextos intraoperatorios. Esta posee efectos fisiológicos característicos en los diferentes sistemas corporales, los cuales son esenciales de conocer a la hora de utilizar este método anestésico. Dichos efectos pueden cobrar principal relevancia en ciertos pacientes según sus requerimientos, comorbilidades o el procedimiento al cual serán sometidos. En esta revisión se destacan los efectos que la anestesia espinal produce en los principales sistemas del organismo. En el sistema nervioso autonómico, los efectos dependerán de la regulación de los sistemas simpático y parasimpático secundaria al nivel de bloqueo, el cual puede ser estimado mediante el nivel de discriminación térmica. El bloqueo simpático producido se traduce en vasodilatación arterial y venosa sistémica, lo que a su vez causa una disminución de la resistencia vascular periférica, retorno venoso, frecuencia cardiaca y contractilidad miocárdica. En cuanto al sistema nervioso central, la anestesia espinal causaría un efecto sedante mediante distintas teorías. En condiciones normales, la función ventilatoria no resulta significativamente afectada, pero podría volverse insuficiente en ciertos pacientes o verse afectada en cuanto a la regulación de la broncodilatación. Debido a la redistribución de flujo, se ve afectada la termorregulación, presentando disminuciones en la temperatura corporal. Otros sistemas que pueden verse afectados por la regulación del sistema nervioso autonómico son el gastrointestinal y el excretor urinario, además de producirse un eventual bloqueo adrenal que llevaría a una menor respuesta metabólica de estrés.

Palabras clave: Anestesia, anestesia regional, anestesia espinal, fisiología. 


\section{Introducción}

a anestesia espinal condiciona efectos característicos en el organismo, siendo en algunos casos similares y en otros diferentes a aquellos de la anestesia general. Estos son fundamentales de conocer a la hora de individualizar la anestesia según las necesidades y comorbilidades de cada paciente, además de guiar la monitorización del periodo perioperatorio. A continuación se describen algunos de los cambios fisiológicos más relevantes producidos en los principales sistemas durante el uso de anestesia espinal.

\section{Efectos fisiológicos}

\section{Sistema nervioso autonómico}

La inervación visceral efectora o motora es distribuida a través del sistema autonómico simpático y parasimpático. Los centros activadores del sistema simpático están localizados en el hipotálamo, bulbo raquídeo y médula espinal. La vía simpática tiene una primera neurona preganglionar, fibras delgadas mielínicas tipo B, cuyo núcleo está en el área gris intermedio lateral de la médula espinal. Se distribuye a través de los nervios espinales T1 a L2 para formar posteriormente el plexo simpático a nivel para y prevertebral donde hace sinapsis con una neurona posganglionar, fibras amielínicas tipo $C$, que se distribuyen a los distintos órganos (Tabla 1). En ocasiones la sinapsis ocurre previo a la llegada al órgano efector mismo, como es el caso de la inervación suprarrenal. Las fibras simpáticas no mantienen una relación estricta con el nivel somático del nervio espinal que acompañan en su emergencia de la columna vertebral y la inervación que ellas proveen. Hay fibras que ascienden o descienden varios niveles antes de hacer sinapsis en el plexo simpático. El sistema simpático, a nivel somático, provee inervación a vasos sanguíneos, glándulas sudoríparas y músculos piloerectores. A nivel visceral provee inervación a vasos sanguíneos, diferentes glándulas secretoras y músculo liso de diferentes órganos. La altura alcanzada por el bloqueo simpático en una anestesia espinal es difícil de determinar; los diferentes métodos con los cuales se ha pretendido medir su altura tienen sesgos que hacen difícil su interpretación[1]. Para Greene, el método más simple y confiable es suponer que, por diámetro (o más bien, distancia internodal) y velocidad de conducción similares, el comportamiento clínico de bloqueo de las fibras simpáticas preganglionares es comparable a las fibras que transmiten sensibilidad térmica. De este modo, medir el nivel de discriminación térmica equivale a medir el nivel de bloqueo simpático. Se presupone que el bloqueo simpático alcanza entre 2 y 6

\begin{tabular}{ll}
\multicolumn{2}{c}{ Tabla 1. Distribución segmentaria de los nervios simpáticos } \\
\hline Raíz de nervio simpático & Segmento correspondiente \\
T 1 & Cabeza \\
T 2 & Cuello \\
T 1 - T 4 & Inervación cardiaca \\
T $3-$ T 6 & Distribución torácica \\
T 7 - T 11 & Distribución abdominal \\
T $12-$ L 2 & Extremidades inferiores \\
\hline
\end{tabular}

metámeros más a cefálico que lo que es posible medir como bloqueo sensitivo doloroso somático[2]. Hay evidencia sugerente de que no ocurre un bloqueo simpático completo aún con espinales altas (más a cefálico que T3)[3].

El sistema parasimpático se distribuye a través de nervios craneales, principalmente el nervio vago, así como también por los nervios espinales sacros (S2-S4). El resultado en una anestesia espinal es que sólo se bloquea el componente sacro del sistema parasimpático, que inerva al colon descendente, recto, vejiga y genitales externos. Las principales acciones efectoras de los sistemas simpático y parasimpático están resumidas en la Tabla 2[4], exponiendo así los efectos logrados mediante la acción de la anestesia espinal en el sistema nervioso autonómico. La simpaticolisis intratecal da cuenta de la mayor parte de los efectos fisiológicos de la anestesia neuroaxial.

\section{Sistema cardiovascular}

El bloqueo simpático producido durante la anestesia espinal induce vasodilatación arterial y venosa, determinantes de los principales cambios de la fisiología cardiovascular durante esta técnica. La vasodilatación arterial es de una magnitud de 15\%-20\% del diámetro de los vasos[1]. La musculatura lisa de los vasos arteriales mantiene un tono intrínseco a pesar de la denervación, pudiendo aumentar la relajación y consiguiente dilatación vascular frente a otros estímulos como hipoxia y metabolitos producto de la isquemia tisular[1].

La dilatación arterial supone una disminución de la resistencia vascular sistémica, cuya intensidad dependerá del área corporal denervada y la compensación vasoconstrictiva de los territorios no afectados. La venodilatación en las áreas sujetas al efecto del bloqueo espinal es prácticamente máxima. La consecuencia es un aumento de la capacitancia con un aumento del débito cardíaco, aunque con hipotensión arterial si este aumento del débito no es suficiente para compensar. Luego, se agrega disminución del retorno venoso, lo que puede llevar a caída del volumen de fin de diástole ventricular, uno de los principales determinantes del débito cardíaco, con progresiva disminución del débito cardíaco si no se activan mecanismos compensatorios.

La contractilidad miocárdica está disminuida por efecto de la caída del retorno venoso y el volumen de fin de diástole ventricular, a través del fenómeno de Frank Starling. La frecuencia cardiaca disminuye a causa de varios reflejos relacionados a un menor retorno venoso. En primer lugar, el nodo sinusal descarga con una periodicidad proporcional al grado de estiramiento de sus células; a menor estiramiento, por menor volumen auricular dado por menor retorno venoso, menor frecuencia de disparos[5]. Este no es un reflejo nervioso, sino una respuesta intrínseca de las células del nodo. Por otro lado, a nivel de la pared de la aurícula se genera una respuesta refleja nerviosa en la cual, al detectar un mayor estiramiento, aumenta el tono simpático y la frecuencia cardiaca; fenómeno conocido como reflejo de Bainbridge[6]. Al disminuir el volumen de retorno y, por lo tanto, la distensión auricular, podemos suponer una caída de la frecuencia cardiaca.

La inervación simpática cardiaca emerge de las raíces T1 a T4. Una anestesia espinal que alcanza estos segmentos bloqueará el efecto simpático directo sobre el corazón. Los efectos predecibles son disminución de la frecuencia cardiaca y fuerza 


\begin{tabular}{|c|c|c|}
\hline Efectos por estimulación simpática & Sistema/órgano & Efectos por estimulación parasimpática \\
\hline Midriasis & Pupila & Miosis \\
\hline Vasoconstricción, discreta secreción & Glándulas salivales & Intensa secreción \\
\hline Vasoconstricción, discreta secreción & Glándulas gastrointestinales & Intensa secreción \\
\hline Intensa suduración & Glándulas sudoriparas & Suduración palma de manos \\
\hline Intensa secreción & Glándulas odoríparas & Sin efecto \\
\hline Constricción & Vasos sanguíneos & Sin efecto \\
\hline Aumenta & Frecuencia cardiaca & Disminuye \\
\hline Positivo & Inotropismos & Negativo \\
\hline Dilatación & Arterias coronarias & Constricción \\
\hline Dilatación & Bronquios & Constricción \\
\hline Disminuye & Motilidad intestinal & Aumenta \\
\hline Aumenta & Tono esfinteres & Disminuye \\
\hline Libera glucosa & Hígado & Discreta síntesis de glicógeno \\
\hline Relajación & Vesícula biliar & Contracción \\
\hline \multirow[t]{2}{*}{ Disminuye secreción renina } & Riñón & Ninguno \\
\hline & Vejiga & \\
\hline Relajación & Músculo detrusor & Contracción \\
\hline Contracción & Trígono vesical & Relajación \\
\hline Aumenta & Metabolismo basal & Ninguno \\
\hline
\end{tabular}

Tabla modificada de Guyton \& Hall W.B[4].

contráctil. La frecuencia no se ve afectada significativamente por la falta de inervación en el paciente sano en reposo, ya que basalmente hay predominio de la inervación parasimpática con baja actividad simpática. La bradicardia de la anestesia espinal normalmente está asociada al bloqueo de fibras cardioaceleradoras. Sin embargo, es posible ver un descenso de los latidos con niveles de anestesia espinal que no alcanzan a bloquear estas fibras, que serían explicados por los mecanismos ya descritos relacionados con la distensión auricular. Aún no es posible explicar claramente la falta de expresión clínica del barorreflejo ante el descenso de la presión arterial con anestesias que no llegan a bloquear las fibras simpáticas cardiacas.

La anestesia raquídea que produce un bloqueo simpático del cuarto al décimo segmento torácico resulta muy eficaz a la hora de suprimir la respuesta simpático-suprarrenal de estrés y la liberación de catecolaminas, renina y vasopresina[7].

\section{Sistema respiratorio}

La función ventilatoria está controlada por el centro respiratorio ubicado a nivel del bulbo raquídeo. En condiciones normales de reposo, sólo la inspiración es un esfuerzo activo, el cual es realizado por el diafragma, que está inervado por el nervio frénico (C2-C5). No es sorprendente, entonces, que la anestesia espinal, en pacientes sin patología agregada, no se asocie a grandes cambios en la mecánica respiratoria[8]. Sin embargo, la función espiratoria es activa y es una función que depende de la musculatura abdominal y la musculatura intercostal interna, la cual es inervada por nervios espinales somá- ticos torácicos. En una anestesia neuroaxial esta musculatura quedará parcialmente denervada dependiendo del nivel máximo alcanzado. Pacientes que requieren toser continuamente por ser hipersecretores o pacientes con limitación obstructiva al flujo aéreo podrían perjudicarse con la anestesia espinal. Por lo tanto, la implicancia del posible deterioro de la función espiratoria es una consideración teórica que debe tenerse en cuenta en la planificación de una anestesia en el contexto del análisis de riesgos y beneficios de los factores involucrados.

La reactividad bronquial está influenciada por el tono simpático que estimula broncodilatación y del tono parasimpático que estimula broncoconstricción. En un bloqueo espinal alto hasta T4, el balance por interrupción del tono simpático y preservación del tono parasimpático podría favorecer cuadros de broncoconstricción[9].

El intercambio gaseoso podría indirectamente verse afectado como compensación a la disminución del retorno venoso. Habitualmente en el intraoperatorio se realiza un aporte de volumen en base a cristaloides, favoreciendo una menor presión coloidosmótica plasmática que, en el paciente susceptible, podría condicionar la aparición de edema pulmonar e interferencia en el intercambio gaseoso[10].

\section{Sistema nervioso central}

La anestesia espinal se ha asociado con disminución del nivel de conciencia (sedación). Esto se ha medido por observación clínica[11], monitores de profundidad anestésica[12] e, indirectamente, por la disminución en los requerimientos de sedan- 
tes intravenosos como midazolam[13] y propofol[14]. Si bien el mecanismo causal no está dilucidado, podría pensarse en la disminución del flujo cerebral consecuente con la hipotensión arterial como posible explicación. No obstante, los mecanismos de autorregulación cerebral en pacientes sanos mantienen el flujo relativamente constante con presiones medias entre 50 $150 \mathrm{mmHg}$. También podría pensarse en el efecto sistémico de algunas drogas o el ascenso rostral intrarraquídeo, sin embargo, esto es poco probable por las dosis usadas y las concentraciones alcanzadas a nivel ventricular. Hay autores que han sugerido que el mecanismo responsable es la disminución de la aferencia sensorial sobre el sistema reticular, resultando en un efecto hipnótico[15].

\section{Sistema gastrointestinal}

El intestino y vísceras intraabdominales están inervados por fibras simpáticas de T6 a L1 y fibras parasimpáticas provenientes tanto del nervio vago como de S2 a S4, por medio de los nervios hipogástricos, para colon descendente y recto. El bloqueo de fibras simpáticas deja sin contrapeso la inervación parasimpática del nervio vago, resultando en un intestino contraído, esfínteres relajados y aumento del peristaltismo, lo que pudiera interferir en algunas cirugías que requieren que el intestino esté relajado e inmóvil. Además, pudiera explicar en parte la incidencia de náuseas y vómitos intraoperatorios asociados a la técnica, principalmente por estímulos (tracción mesentérica, estímulos peritoneales, etc.) conducidos por fibras nerviosas integradas por medio del nervio vago, que habitualmente no se bloquean. La función hepática no se ve alterada y el flujo sanguíneo visceral podría encontrarse aumentado o disminuido.

\section{Riñón y vejiga}

Clínicamente la función renal durante una anestesia intratecal tiene cambios de baja magnitud en el sujeto sano. Teóricamente, sin embargo, existen múltiples mecanismos por los cuales podría verse afectada. La caída de la presión arterial sistémica podría disminuir el flujo renal. La inervación simpática renal (T10-L2) y las catecolaminas circulantes tienen varios efectos en el riñón, entre ellos: vasoconstricción de arteriolas renales con disminución de la filtración glomerular; aumento de la reabsorción de sodio, agua tubular y aumento de la liberación de renina. La presencia de mecanismos de autorregulación y la reserva renal mantienen la función renal normal a pesar de los cambios anteriores. Así, a pesar de que una disminución previsible del flujo sanguíneo renal acompaña al bloqueo neuroaxial, esta disminución es de poca importancia fisiológica[16]

Por su parte, la vejiga debe acumular la orina producida y posteriormente vaciarla. Está inervada por fibras sensitivas con receptores de estiramiento de la pared vesical. También recibe inervación parasimpática motora que produce contracción del músculo detrusor. Ambas se encuentran en nervios pélvicos dependientes de segmentos sacros (S2-S4). Además, de la contracción del músculo detrusor, durante la micción debe sobrepasarse el tono natural del esfínter uretral interno y la relajación del esfínter uretral externo de control voluntario inervado por fibras somáticas del nervio pudendo (S2-S4). La inervación sacra de la vejiga y el reflejo de micción están precozmente expuestos al bloqueo nervioso de la anestesia espinal. El bloqueo de las fibras sensitivas impide la señal de distensión y el bloqueo parasimpático, disminuye el tono contráctil del músculo detrusor facilitando la acumulación de orina y distensión de la vejiga. La inhibición prolongada del detrusor puede llevar a la sobredistensión vesical y daño teórico de la capacidad de contracción posterior[17].

\section{Termorregulación}

Durante la anestesia espinal hay una disminución de la temperatura corporal central, por redistribución del calor del cuerpo entre compartimentos internos y periféricos afectados por la vasodilatación cutánea, consecuencia del bloqueo simpático. Esto favorece un mayor flujo de sangre hacia estas zonas vasodilatadas, donde se mezcla con sangre que se encuentra habitualmente a menor temperatura y vuelve al sistema central. La magnitud del descenso es, la mayoría de las veces, menor que durante la anestesia general, pero puede ser igual o mayor dependiendo de la magnitud y duración del acto quirúrgico. Durante la anestesia general se alcanza una meseta hipotérmica producto de la fijación de una nueva temperatura termorreguladora. En cambio, bajo la anestesia neuroaxial no se produce esta meseta sino un descenso mantenido[18], cuya pendiente dependerá de la duración de la cirugía y la superficie corporal descubierta. El bloqueo simpático y motor conlleva a una incapacidad de activar mecanismos compensadores de vasoconstricción y calofríos en los territorios bloqueados. Las áreas no anestesiadas pueden ser insuficientes para mantener la temperatura corporal.

\section{Respuesta metabólica y estrés}

La respuesta metabólica y de estrés medida por niveles de catecolaminas circulantes, alza de glicemia y cortisol plasmático, están disminuidos durante la anestesia regional[19]. El mecanismo principal está dado por el bloqueo eferente adrenal y, por ende, dura mientras exista bloqueo espinal. El hecho que sólo sea una disminución y no una completa abolición de la respuesta[20] presupone mecanismos probablemente locales del sitio quirúrgico que por vías humorales sean capaz de gatillar parcialmente la respuesta metabólica.

\section{Referencias}

1. Greene NM, Brull SJ. Physiology of Spinal Anesthesia. 4th ed. Baltimore: Williams \& Wilkins; 1993. 387 p.

2. Greene NM. A new look at sympathetic denervation during spinal anesthesia. Vol. 65, Anesthesiology. 1986.

3. Stevens RA, Frey K, Liu SS, Kao TC, Mikat-Stevens M, Beardsley $D$, et al. Sympathetic block during spinal anesthesia in volunteers using lidocaine, tetracaine, and bupivacaine. Reg Anesth. 1997;22(4).

4. Guyton AC, Hall JE. Textbook of medical physiology. 11th ed. Elsevier, editor. Philadelphia; 2006. 754 p.

5. Cooper PJ, Kohl P. Species- and preparation-dependence of stretch effects on sino-atrial node pacemaking. In: Annals of the New York Academy of Sciences. 2005.

6. Guyton AC, Hall JE. Textbook of medical physiology. 11th ed. Elsevier Saunders, editor. Philadelphia; 2006. 1116 p. 
7. Mcllroy D, Sladen RN. Fisiología, fisiopatología y farmacología renales. In: Miller R, Eriksson L, Fleisher L, Wiener-Kronish J, Cohen $N$, Young W, editors. Miller Anestesia. 8th ed. Philadelphia: Saunders; 2015. p. 545-89.

8. Von Ungern-Sternberg BS, Regli A, Reber A, Schneider MC. Comparison of perioperative spirometric data following spinal or general anaesthesia in normal-weight and overweight gynaecological patients. Acta Anaesthesiol Scand. 2005;49(7).

9. Capelozzi M, Arantes FM, Paiva PSO, Capelozzi VL, Martins MA. Spinal anesthesia increases pulmonary responsiveness to methacholine in guinea pigs. Anesth Analg. 1998;87(4).

10. Silverstein JH, Rooke A, Reves JG, McLeskey CH. Geratric Anesthesiology. 2nd ed. Springer, editor. New York; 2007. 440 p.

11. Gentili M, Chau Huu P, Enel D, Hollande J, Bonnet F. Sedation depends on the level of sensory block induced by spinal anaesthesia. Br J Anaesth. 1998;81(6).

12. Morley AP, Chung DC, Wong ASY, Short TG. The sedative and electroencephalographic effects of regional anaesthesia. Anaesthesia. 2000;55(9).

13. Ben-David B, Vaida S, Gaitini L. The influence of high spinal anesthesia on sensitivity to midazolam sedation. Anesth Analg.
$1995 ; 81(3)$

14. Ozkan-Seyhan $T$, Sungur MO, Senturk E, Karadeniz M, Basel A, Senturk M, et al. BIS guided sedation with propofol during spinal anaesthesia: Influence of anaesthetic level on sedation requirement. Br J Anaesth. 2006;96(5)

15. Pollock JE, Neal JM, Liu SS, Burkhead D, Polissar N. Sedation during spinal anesthesia. Anesthesiology. 2000;93(3).

16. Brull $R$, Macfarlane AJR, Chan VWS. Anestesia intradural, epidural y caudal. In: Miller R, Eriksson L, Fleisher L, Wiener-Kronish J, Cohen N, Young W, editors. Miller Anestesia. 8th ed. Philadelphia: Saunders; 2015. p. 1683-720.

17. Mulroy MF, Salinas F V., Larkin KL, Polissar NL. Ambulatory surgery patients may be discharged before voiding after short-acting spinal and epidural anesthesia. Anesthesiology. 2002;97(2).

18. Sessler DI. Perioperative heat balance. Vol. 92, Anesthesiology. 2000.

19. Desborough JP. The stress response to trauma and surgery. $\mathrm{Br} J$ Anaesth. 2000;85(1).

20. Oehmke MJ, Podranski T, Mann M, Frickey N, Kuhn DFM, Hempelmann G. Perioperative concentrations of catecholamines in the cerebrospinal fluid and plasma during spinal anesthesia. Acta Anaesthesiol Scand. 2008;52(4). 